\title{
Mutation (variation) databases and registries: a rationale for coordination of efforts
}

\author{
Arleen D. Auerbach, John Burn, Jean-Jacques Cassiman, Mireille Claustres, \\ Richard G. H. Cotton, Garry Cutting, Johan T. den Dunnen, Mona El-Ruby, \\ Aida Falcon Vargas, Marc S. Greenblatt, Finlay Macrae, Yoichi Matsubara, \\ David L. Rimoin, Mauno Vihinen and Christine Van Broeckhoven
}

The importance of gene- or locus-specific databases (LSDBs) has recently been extolled in this journal (The case for locus-specific databases. Nature Reviews Genetics 12, 378-379 (2011)) $)^{1}$. Here we argue that coordination of international efforts for developing comprehensive mutation databases and patient phenotype registries is essential for optimal genetic health care.

Well-funded international efforts for setting up mutation databases or registries are crucial for several reasons. Many variants that are found during clinical testing worldwide are not submitted to databases, where they could form an important resource for patient care. Many laboratories and clinicians do not have the capacity or incentive to submit data to databases. This is especially the case in developing countries owing mainly to technical insufficiency, lack of public awareness, lack of international communications, the absence of the concept of DNA biobanking, national authority restrictions and lack of translation from original languages to English.

The Human Variome Project (HVP) was initiated to facilitate the collection of all variants in all genes from all countries and to include annotation of these variants for pathogenicity and relevance to clinical medicine $\mathrm{e}^{2}$. It was established at a meeting in 2006 that was attended by representatives of the World Health Organization (WHO), the United Nations Educational, Scientific and Cultural Organization (UNESCO), the Organisation for Economic Co-operation and Development (OECD), the European Commission, March of Dimes, the US National Center for Biotechnology Information, the European Bioinformatics Institute (EBI) and 30 countries $^{3}$. The third HVP meeting at UNESCO Headquarters in 2010 allowed the election of an International Scientific Advisory Committee and affirmation of a Roadmap ${ }^{4}$. Most recently, China has committed $\$ 300$ million to the project ${ }^{5}$, and UNESCO has awarded the HVP the status of 'NGO in operational relations with UNESCO'. Many working groups are establishing standards for collecting, presenting and sharing variation information.

Registries for inherited diseases have been developed in some countries, especially where therapies are available (for example, see REF. 6). Recently, there has been a call for global registries of rare diseases (more than $80 \%$ of which are genetic) $)^{7,8}$. Most recently, the US National Institutes of Health and the European Commission have developed the International Rare Diseases Research Consortium (IRDiRC) 9 .

These two initiatives, the HVP and IRDiRC, have been developing essentially independently and in parallel. The HVP was driven by clinicians and laboratories wishing to have access to complete disease-associated variation information to support diagnostic advice and to facilitate the publication of novel mutations of interest. Recently, the focus has moved to collecting all mutations in all genes from all countries ${ }^{10}$ as a means of assisting the interpretation of functional effects of genetic variations. The IRDiRC has been driven by patient groups who are anxious to achieve therapy for their families' diseases and to recruit cohorts for clinical trials in registries.

Practically, the promised funds from China in support of the HVP will allow 5,000 databases to be properly set up. If the decision is to set up these databases as both mutation and patient registries, this will assist both initiatives and avoid duplication.

Each group has their own networks, methodology, experts, data content and specifications. It would seem wasteful if two parallel systems were developed when many data are in common and when global reach is needed by both. In the case of the HVP, key components that are in place are a federated model, forums for sharing experiences, development of best informatics practices that are relevant to the task, and leadership.
Clearly in the case of the IRDiRC, the key components are model registries, Orphanet experience and Genetic Alliance experience.

Future generations will pay the price for a failure to establish a joint international approach to the recording of and provision of access to human molecular variation, as such access is the most important step in approaching the diagnosis, and thus prevention, of inherited disorders.

\section{The authors are all members of the International Scientific Advisory Committee of the Human Variome Project.}

Arleen D. Auerbach is at The Rockefeller University, New York, New York 10065, USA.

John Burn is at the Institute of Genetic Medicine, Newcastle University, International Centre for Life, Newcastle upon Tyne NE1 3BZ, UK

Jean-Jacques Cassiman is at the Center for Human Genetics, Forensic Medicine Campus, Katholieke Universiteit Leuven, Leuven, Belgium.

Mireille Claustres is at the Institut of Universitaire Clinical Research, University Hospital of Montpellier Montpellier 34093, France

Richard G. H. Cotton is at the Human Variome Project, University of Melbourne, Melbourne, Australia.

Garry Cutting is at the Johns Hopkins School of Medicine, Baltimore, Maryland, USA

Johan T. den Dunnen is at Leiden University Medical Center, 2333 ZA Leiden, The Netherlands.

Mona El-Ruby is at the National Research Centre, Dokki, Cairo 12311, Egypt.

Aida Falcon Vargas is at the Centro Clínico Profesional Caracas, Anexo Hospital de Clínicas Caracas, San Bernardino, Caracas, Venezuela.

Marc Greenblatt is at the University of Vermont, Burlington, Vermont 05405, USA

Finlay Macrae is at The Royal Melbourne Hospital, Parkville 3050, Melbourne, Victoria, Australia.

Yoichi Matsubara is at Tohoku University School of Medicine, Sendai, Miyagi 980-8574, Japan.

David Rimoin is at the Cedars-Sinai Medical Center, Los Angeles, California 90048, USA.

Mauno Vihinen is at the University of Tampere and BioMediTech, Tampere, Finland

Christine Van Broeckhoven is at the VIB Department of Molecular Genetics, University of Antwerp, 2610 Antwerp, Belgium

Correspondence to A.D.A e-mail:auerbac@mail.rockefeller.edu

doi: 10.1038/nrg3011-c1 Published online 25 October 2011

Samuels, M. E. \& Rouleau, G. A. The case for locusspecific databases. Nature Rev. Genet. 12, 378-379 (2011).

Anonymous. What is the Human Variome Project? Nature Genet. 39, 423 (2007).

Cotton, R. G. et al. Recommendations of the 2006 Human Variome Project meeting. Nature Genet. 39 , 433-436 (2007).

Kohonen-Corish, M. R. J. et al. How to catch all those mutations - the report of the third Human Variome Project Meeting, UNESCO Paris, May 2010. Hum. Mutat. 31, 1374-1381 (2010). 
5. Cyranoski, D. China spurs quest for human variome Nature 469, 455 (2011).

6. Byrne, B. J. et al. Pompe disease: design, methodology, and early findings from the Pompe registry. Mol. Genet. Metab.103,1-11 (2011).

7. Rubinstein, Y. R. et al. Creating a global rare disease patient registry linked to a rare diseases biorepository database: Rare Disease-HUB (RD-HUB). Contemp.

Clin. Trials. 31, 394-404 (2010).
8. Forrest, C. B. et al. The case for a global rare disease registry. Lancet 377, 1057-1059 (2011).

9 Abbott, A. Rare-disease project has global ambitions. Nature 472, 17 (2011).

10. Cotton, R. G. et al. Capturing all disease-causing mutations for clinical and research use: toward an effortless system for the Human Variome Project. Genet. Med. 11, 843-849 (2009).
Competing interests statement

The authors declare no competing financial interests.

FURTHER INFORMATION

The Human Genome Variation Society: www.hgvs.org

The Human Variome Project: www.humanvariomeproject.org

ALL LINKS ARE ACTIVE IN THE ONLINE PDF 